\title{
Effect of Milling Time on Phase Composition and Microstructure of Ti-Sn-C Powder
}

\section{Jin Chenggong}

Beihua University Engineering Training Center, Jilin, 132021, China

Keywords: mechanical alloying, Ti2SnC, Sn whisker

\begin{abstract}
The Ti2SnC conductive ceramic materials were synthesized by using Ti, Sn and C as the reaction materials. The effects of milling time on the phase composition and microstructure of Ti-Sn-C mechanical alloying products were studied. The results show that the Ti2SnC typical lamellar structure is observed by the XRD pattern of Ti2SnC as the main peak when the milling time is constant. When the time is close to the critical point of its self-propagating reaction, a large number of Sn whiskers are grown, and the analysis of the elemental Sn is refined by ball milling to produce a strong plastic deformation.
\end{abstract}

\section{Introduction}

$\mathrm{Ti}_{2} \mathrm{SnC}$ is a new type of conductive ceramic material, available molecular formula $\mathrm{Mn}+1 \mathrm{AXn}$, with a similar structure are Ti3GeC2 and Ti3AlC2 and other compounds [1]. The presence of metal bonds, strong covalent bonds and intermolecular bonds of the transition metal octahedrons in the structure of this compound is such a special structure that imparts the overall properties of metals and ceramics [2-3]. Ti2SnC has good electrical conductivity, good heat transfer performance and good plasticity at room temperature, and can be used for mechanical processing. It can also be used to make complex structural parts. At the same time, it has the advantages of ceramic oxidation resistance, heat shock resistance and high fracture toughness. The Such composites generally have a lower coefficient of friction.

According to the isothermal cross-sectional phase diagram of Ti-Sn-C at $1200{ }^{\circ} \mathrm{C}$, it is found that $\mathrm{Ti}_{2} \mathrm{SnC}$ is stable only in a very small area. When the composition ratio is slightly different, $\mathrm{Ti}_{6} \mathrm{Sn} 5$, $\mathrm{Ti}_{5} \mathrm{Sn} 3, \mathrm{Ti}_{2} \mathrm{Sn}$ and TiCX are formed. Purity of $\mathrm{Ti}_{2} \mathrm{SnC}$. At present, the synthesis of more successful methods of pressure sintering, liquid-solid reaction method, hot isostatic pressing method [4-6]. Yu [7] and so on Ti / Sn / C element powder by 211 molar ratio of ingredients, at a certain temperature self-propagating reaction, high purity $\mathrm{Ti}_{2} \mathrm{SnC}$ powder. But the above methods are high temperature synthesis, the experimental process is relatively complex. In this paper, $\mathrm{Ti}_{2} \mathrm{SnC}$ powder was synthesized by mechanical alloying method [8-9], and the influence of milling time on the morphology and phase composition of the powder was studied. And obtain high performance and excellent performance of ceramic materials.

\section{Experimental Method}

A high purity $\mathrm{Ti}_{2} \mathrm{SnC}$ ceramic powder was prepared by mechanical alloying method using $\mathrm{Ti}$ powder, Sn powder and $C$ powder as the reaction raw material. Ball ratio of 10: 1 , ball diameter of $12 \mathrm{~mm}$, milling time of $2.5 \sim 10 \mathrm{~h}$. (002) diffraction peak of $\mathrm{Ti}_{2} \mathrm{SnC}$ was selected as the characteristic peak in the Ti-Sn-C ternary system by using the D/Max2500PC X-ray diffractometer to analyze the powder after ball milling ( $\mathrm{Cu}$ target, $\mathrm{K} \alpha) 2 \theta=12.95^{\circ}$; the (111) peak of TiC is its characteristic peak, $2 \theta=35.9^{\circ}$. The morphology of the powder was observed by JSM-5600LV scanning electron microscopy (SEM). Prior to TEM detection, the $\mathrm{Ti}_{2} \mathrm{SnC}$ powder was dissolved in alcohol as a solvent and subjected to sonication to disperse the particles as much as possible. 


\section{Experimental Results and Analysis}

Effect of Mechanical Ball Milling Time on Morphology and Phase Transition of Mixed Powders
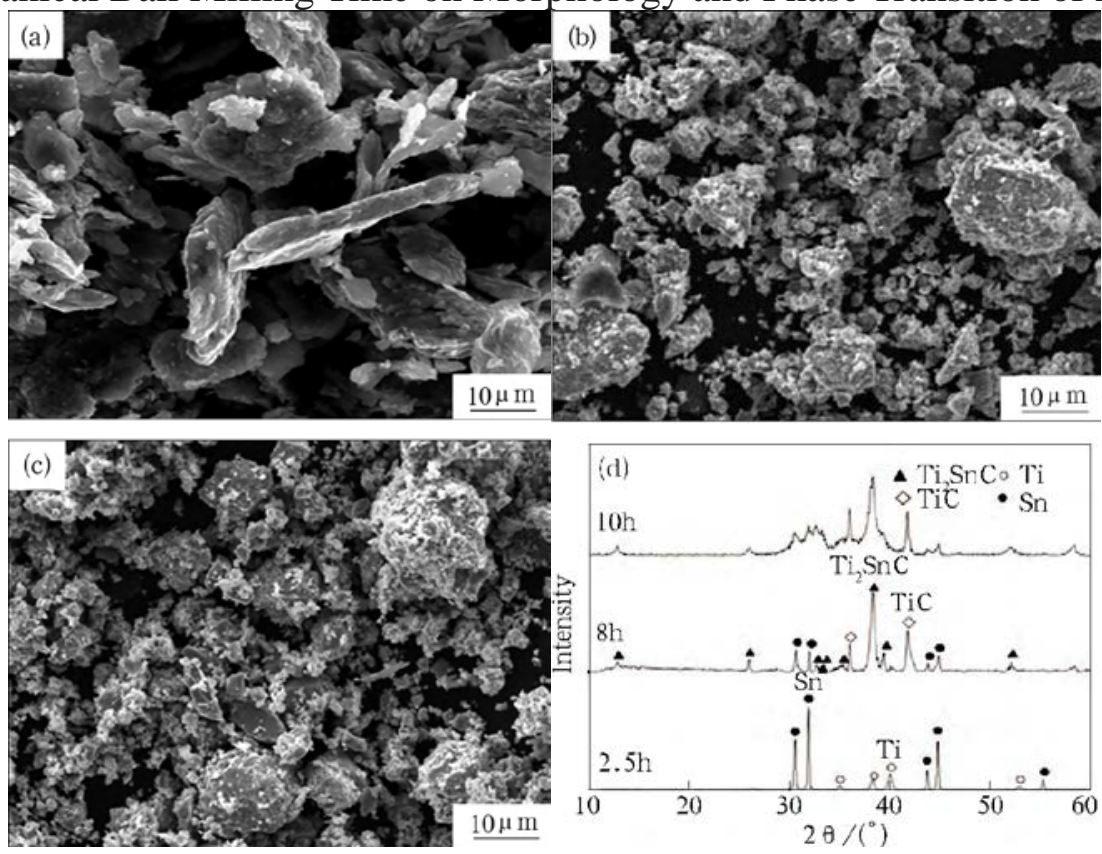

Fig.1 SEM micrograph and XRD patterns of the powders after different milling times (a)2.5h, (b)8h, (c) $10 \mathrm{~h}$

Fig.1 shows the morphology and phase composition of the mixed powders obtained by mechanical alloying at different milling times. Fig.1 (a) shows the microstructure of the mixed powder obtained by milling for $2.5 \mathrm{~h}$. It is found that the microstructure of the mixture is composed of particles of uneven size, and the XRD pattern of the mixed powder is shown in Fig. 1 (d). In the XRD pattern only diffraction peaks of $\mathrm{Ti}$ and $\mathrm{Sn}$, indicating that the raw material powder at this time did not react to generate new material, but powder ball mill grinding ball after multiple collisions, more refined grain size, specific surface area increases, has been facilitated for the subsequent reaction the role of. $\mathrm{C}$ is an amorphous element, so it is not shown in the XRD pattern. When the milling time is extended to $8 \mathrm{~h}$ as shown in Fig. 1 (b), the microstructure of the structure changes from granular to $\mathrm{Ti}_{2} \mathrm{SnC}$, with a slight agglomeration phenomenon in which the diffraction peak of Ti disappears in the XRD pattern, $\mathrm{Ti}_{2} \mathrm{SnC}$ and TiC diffraction peaks appeared, and $\mathrm{Ti}_{2} \mathrm{SnC}_{\mathrm{C}}$ was the main peak, indicating that the mixed powder was reacted with $\mathrm{Ti}_{2} \mathrm{SnC}$ as the main phase under the process of prolonging the milling time. When the milling time is extended to $10 \mathrm{~h}$ as shown in Fig. 1 (c), the agglomeration of the structure is more serious, and the intensity of the Ti2SnC diffraction peak in the XRD spectrum also begins to decrease, indicating that the system energy is too large, resulting in $\mathrm{Ti}_{2} \mathrm{SnC}$ decomposition The In the process of milling, with the milling time prolonged, the powder particles continue to be refined, the final powder particles become nano-scale, this time the surface atomic ratio of the powder, the specific surface area, the surface energy, energy instability Of the state, and thus refine the particles tend to gather together, so the emergence of the above-mentioned serious agglomeration.

Fig. 2 is the raw material powder ball 8 h, placed for a period of time after the powder obtained micro-morphology and phase composition. In Fig. 2 (a) shows that a large number of needle-like whiskers appear in the structure of the mixed powder. To determine the phase composition of whiskers. The powder was subjected to XRD diffraction analysis. It can be seen from Fig. 2 (b) that the diffraction peak of elemental $\mathrm{Sn}$ is the main peak and the diffraction peak of element Ti is weak. In addition, the whiskers were not found in the powder with too short milling time, and the whiskers in the synthetic ceramic phase had less or no whiskers, and the whiskers appeared in the ball milling time close to the critical point of the self-propagating reaction. in. Analysis of the principle of forming Sn whiskers: Element $\mathrm{Sn}$ in the mechanical alloying process by the continuous impact of 
the ball, the particle size of the refinement, and a large number of plastic deformation occurred, the powder particles accumulated stress and a certain amount of heat, the recrystallization temperature of Sn is very low. During the process of placing, the recrystallization of the elemental Sn occurs with rapid directional growth to form whiskers. The stress during the mechanical alloying provides the main driving force for the growth of tin whiskers.

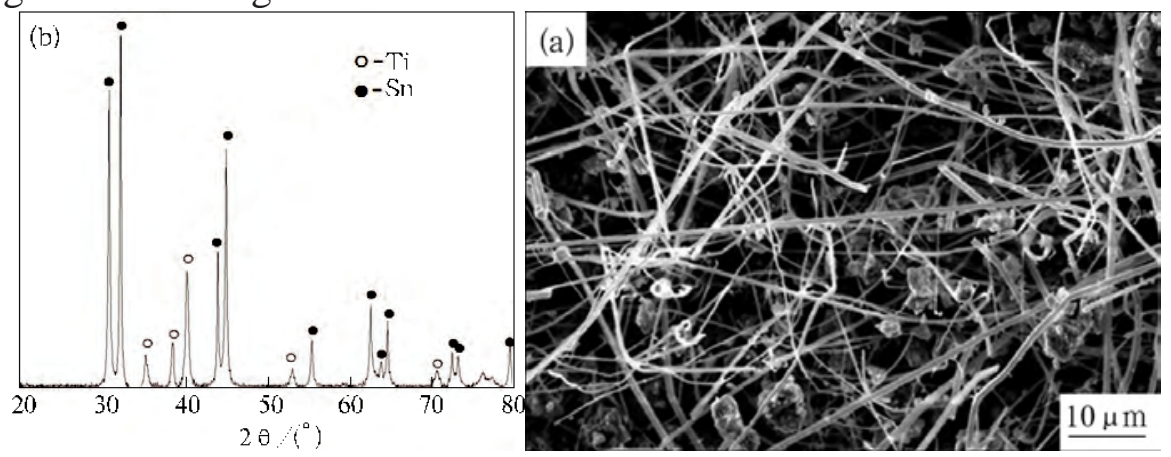

Fig.2 SEM micrograph and XRD patterns of the whiskers

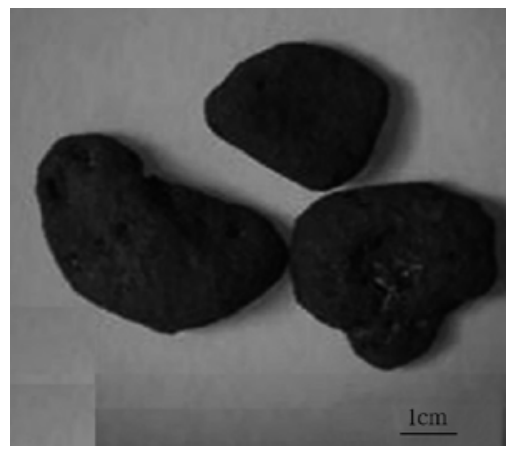

Fig. 3 The coarse granule formed during the mechanical alloying

In the experimental results detected, found some size of about $0.5 \sim 1 \mathrm{~cm}$ block, as shown in Figure 3. These blocks are grayish black, hard texture, polished with sandpaper, and the surface of the block has honeycomb holes. The reason for the formation of this hole may be: in the process of mechanical alloying, Ti2SnC and $\mathrm{TiC}$ are all exothermic reactions. The release of heat causes the local temperature of the whole system to rise to the melting point of some kind of reactant, even Gasification and with the rapid solidification process of particles discharged, resulting in some pores. In the process of mechanical alloying, this process of reacting from reactants in such a short period of time to produce products is difficult to explain with the traditional diffusion theory, which is typical of the mechanical induced self-propagating reaction (MSR) feature [10].

\section{Conclusion}

In this paper, $\mathrm{Ti}_{2} \mathrm{SnC}$ ceramic powder was synthesized by mechanical alloying process using $\mathrm{Ti}$, $\mathrm{Sn}$ and $\mathrm{C}$ powder as the reaction materials. The effects of milling time on the phase composition and microstructure of Ti-Sn-C powder were studied. With the increase of milling time, the powder particles are constantly refined and the powder form gradually changes from granular to lamellar, and the ball is pulverized for $8 \mathrm{~h}$, and the ceramic powder material with $\mathrm{Ti}_{2} \mathrm{SnC}$ as the main phase appears. The milling time increased to $10 \mathrm{~h}$, the lamellar particles disappeared completely and transformed into granular particles containing $\mathrm{Ti}_{2} \mathrm{SnC}$. When the milling time is close to the critical point $(8 \mathrm{~h})$ of the self-propagating reaction, a large number of whiskers are grown during the powdering process, and the result of rapid growth of the recrystallized $\mathrm{Sn}$ is determined. The stress is the main driving force for the growth of Sn whiskers.

\section{References}

[1] Barsoum M W,Yaroschuk G . Fabrication and characterization of M2SnC (M=Ti, Zr, Hf and Nb) 
[J]. Scripta Materialia, 1997, 37(10):1583-1591.

[2] Jeitschko W, Nowotny H, Benesovsky F.Kohlen-stoffhaltige ternare Verbindungen (H-Phase) [J]. MonatschChem, 1963,94:672-676.

[3] Barsoum M W.The MN+1AXN phases: a new class of solids; thermodynamically stable nanolaminates [J]. ProgSolid St Chem,2000,28:201-281.

[4] H.Vincent, C.Vincent, B.F.Mentzenetal. Chemical interaction between carbon and titanium dissolved in liquid tin: crystal structure and reactivity of Ti2SnC with $\mathrm{Al}$ [J]. Materials Science and Engineering,1998,256:83-91.

[5] Li Shibo, Bei Guoping, Zhai Hongxiang, et al. Synthesis of Ti2SnC from Ti/Sn/TiC powder mixtures by pressureless sintering techniquee[J]. Letters, 2006(60) Materials:3530-3532.

[6] LiShibo, BeiGuoping, ZhaiHongxiang, etal. Synthesisof Ti2SnC at low-tempera-tureusing mechanically activated sintering process[J]. Materials Science and Engin-eering,2007,457:282-286.

[7] Yuxin Li,Peikang Bai,Bin of C/Ti ratio on self-propagating high-temperature synthesis reaction of Sn-Ti-C system for fabricating Ti2SnC ternary compounds [J]. Journal of Alloys and Compounds.1 September 2011,509(35): L328-L330.

[8] Kuang Xuecheng,Ning Xiaorong Mechanical alloying method for the preparation of BI-TE based thermoelectric materials and its thermoelectric properties [J]. China Ceramics, 2009,45: 35-39.

[9] Wang Qingxue, Zhang Lianmeng Mechanical alloying-new solid-state non-equilibrium processing technology [J]. Chinese Ceramics, 2002,2 (38): 36-39.

[10] Takacs L.Ball milling-induced combustion in powder mixtures containing Titanium Zirconium, or Hafnium [J]. J. Solid State Chem.,1996,125:75-84. 